\title{
Classical stability and quantum instability of black-hole Cauchy horizons
}

\author{
Dragoljub Marković \\ Department of Physics, University of British Columbia, Vancouver, British Columbia, Canada V6T $1 Z 1$ \\ Eric Poisson* \\ Theoretical Astrophysics, California Institute of Technology, Pasadena, California 91125
}

(Draft October 17, 2018; resubmitted version)

\begin{abstract}
For a certain region of the parameter space $\{M, e, \Lambda\}$, the Cauchy horizon of a (charged) black hole residing in de Sitter space is classically stable to gravitational perturbations. This implies that, when left to its own devices, classical theory is unable to retain full predictive power: the evolution of physical fields beyond the Cauchy horizon is not uniquely determined by the initial conditions. In this paper we argue that the Cauchy horizon of a Reissner-Nordström-de Sitter black hole must always be unstable quantum mechanically.

PACS numbers: 04.70.Dy; 04.62.+v
\end{abstract}

It is well known that general relativity admits a well posed initial value formulation [1]. This implies that, given suitable initial data on a spacelike hypersurface $\Sigma$, the solution to the Einstein equations is uniquely determined (up to diffeomorphisms) everywhere within the domain of dependence of the initial surface, $D(\Sigma)$. For some spacetimes however, $D(\Sigma)$ fails to cover the whole manifold. The boundary of $D(\Sigma)$ is known as the Cauchy horizon of the initial surface. In spacetimes with Cauchy horizons, the field equations lose their ability to completely determine the future evolution of the gravitational field; predictability is lost at the Cauchy horizon.

A particular example of a spacetime with Cauchy horizon, which shall form the arena of this Letter, is that of a black hole possessing either electric charge or angular momentum. For simplicity, we shall restrict our attention to the case of a non-rotating, spherically symmetric, charged black hole, as described by the ReissnerNordström solution. This spacetime is well-known to contain a Cauchy horizon [1], which is coincident with the black-hole inner apparent horizon.

The Cauchy horizon of the Reissner-Nordström spacetime is known to be unstable to time-dependent perturbations [2]. Physically, the instability has to do with the fact that the causal past of the Cauchy horizon contains all of the spacetime external to the black hole. Physical effects occurring outside appear highly blueshifted as seen by internal observers near the Cauchy horizon; the blueshift becomes infinite at the Cauchy horizon.

The gravitational field near the Cauchy horizon of a perturbed Reissner-Nordström black hole has been the subject of vigorous investigation [3]. It appears that in the perturbed spacetime, the Cauchy horizon is replaced by a null curvature singularity. This singularity is characterized not by a collapse to zero area, but by an unbounded increase of the internal mass function [3].

This Cauchy-horizon instability offers an interesting way out of the loss-of-predictability problem. That the Cauchy horizon becomes a curvature singularity implies that the classical evolution of the spacetime cannot proceed beyond the Cauchy horizon. In this sense, the predictive power of the theory stays intact. Of course, the classical theory becomes invalid in the vicinity of the singularity, where quantum effects are presumably important [4]. The point is that classical theory must break down before the Cauchy horizon is encountered.

It is striking that, in the case of black-hole spacetimes and within the realm of classical physics, the Cauchyhorizon instability does not serve as a universal mechanism capable of restoring the full predictive power of the field equations. Indeed, black-hole spacetimes exist for which the Cauchy horizon is classically stable. While this is not generally possible if the black hole resides in asymptotically-flat space, stability can be arranged if the black hole is immersed in de Sitter space.

In this Letter, we show that once quantum physics is invoked, the full predictive power of general relativity can be restored: even when it is classically stable, the Cauchy horizon cannot be stable quantum mechanically (excluding possibly a set of measure zero of spacetimes, as we shall explain). Instability is caused by the divergence of the renormalized expectation value of the stress-energy tensor associated with quantized matter fields.

That quantum effects are needed to prevent a loss of predictability at the Cauchy horizon is most intriguing. In this respect, the physics of black-hole Cauchy horizons is remarkably similar to that of chronology horizons [5].

We begin with a brief review of the ReissnerNordström-de Sitter (RNdeS) spacetime [6].

The solution to the Einstein-Maxwell equations (with cosmological constant $\Lambda$ ) representing a charged black hole in de Sitter space is given by

$$
\begin{aligned}
d s^{2} & =-f d v^{2}+2 d v d r+r^{2}\left(d \theta^{2}+\sin ^{2} \theta d \phi^{2}\right) \\
f & =1-2 M / r+e^{2} / r^{2}-\frac{1}{3} \Lambda r^{2}
\end{aligned}
$$

Here, $v$ is a null coordinate which is constant along radial $(d \theta=d \phi=0)$, ingoing ( $r$ decreasing) null geodesics; $M$ 
and $e$ are, respectively, the mass and charge of the black hole. We use units in which $G=c=1$.

The RNdeS spacetime possesses three types of horizons. The cosmological horizon is located at $r=r_{c}$, and the black-hole outer horizon at $r=r_{e}$; the inner horizon $\left(r=r_{i}\right)$ is also a Cauchy horizon for any external spacelike hypersurface. The roots $r_{i}<r_{e}<r_{c}$ are determined by solving the quartic $f=0$; the fourth root is unphysical. The surface gravity of the horizon $r=r_{j}$ is given by $\kappa_{j}=\frac{1}{2}\left|f^{\prime}\left(r_{j}\right)\right|$; here and throughout, a prime denotes differentiation with respect to the argument.

We now introduce three observers in the RNdeS spacetime (Fig. 1). $\mathcal{C}$ is a free-falling observer who crosses the future cosmological horizon; $r$ increases along $\mathcal{C}$ 's world line. $\mathcal{C}^{\prime}$ is a static observer, located just inside the cosmological horizon; along $\mathcal{C}^{\prime}$ 's world line, $r=r_{c}(1-\epsilon)$, where $\epsilon$ is a small, positive constant. Finally, $\mathcal{I}$ is a free-falling observer who crosses the Cauchy horizon; $r$ decreases along $\mathcal{I}$ 's world line.

Mellor and Moss [7] have carried out a classical perturbation analysis for the Reissner-Nordström-de Sitter spacetime, and have shown that a region of the parameter space $\{M, e, \Lambda\}$ exists for which the Cauchy horizon is stable. This region is defined by the inequality

$$
\kappa_{i} \leq \kappa_{c},
$$

which was first written down by Brady and Poisson [8]. Recently, Chambers and Moss [9] have shown that Eq. (2) also implies stability for a rotating, charged black hole residing in de Sitter space.

The condition (2) for classical stability can be motivated simply. The following discussion, borrowed from Ref. [8], will help us understand the differences between the classical and quantum-mechanical stability analyses.

We consider, in the fixed RNdeS background, a test distribution of non-interacting massless particles, in the continuum limit. The particles originate from the cosmological region, move radially inward along curves $v=$ const., and eventually fall into the black hole. They are described by the stress-energy tensor

$$
T_{\alpha \beta}=\left[L(v) / 4 \pi r^{2}\right]\left(\partial_{\alpha} v\right)\left(\partial_{\beta} v\right) .
$$

We suppose that $L(v)$ has support all the way to $v=\infty$. To take into account the coordinate singularity at the future cosmological horizon, we let $L(v)=K e^{-2 \kappa_{c} v}$, where $K$ is a constant, in the limit $v \rightarrow \infty$. A simple calculation then shows that observer $\mathcal{C}$ measures an energy density $\rho_{\mathcal{C}}$ that is everywhere finite and non-vanishing [10]. Observer $\mathcal{C}^{\prime}$, on the other hand, measures an energy density $\rho_{\mathcal{C}^{\prime}}$ that vanishes in the limit $\epsilon \rightarrow 0$; for fixed $\epsilon$, $\rho_{\mathcal{C}^{\prime}} \propto f^{-1} e^{-2 \kappa_{c} v}$.

Next, we consider the influx as measured by observer $\mathcal{I}$. The energy density is now given by

$$
\rho_{\mathcal{I}}=\left(\tilde{E}^{2} K / 4 \pi r_{i}^{2}\right) e^{-2\left(\kappa_{c}-\kappa_{i}\right) v},
$$

where $\tilde{E} \equiv-u_{v}$ is a constant. We see that $\rho_{\mathcal{I}}$ is redshifted by the cosmological horizon, and blueshifted by the Cauchy horizon. It is this (infinite) blueshift which tends to produce an instability. Nevertheless, $\rho_{\mathcal{I}}$ stays bounded when Eq. (2) is satisfied, and the Cauchy horizon is then classically stable.

We now turn to the question of quantum stability. We assume that quantum fields exist in the RNdeS spacetime, and we seek to examine the behavior of $\left\langle T_{\alpha \beta}\right\rangle$, the renormalized expectation value of their stress-energy tensor, near the Cauchy horizon.

To calculate $\left\langle T_{\alpha \beta}\right\rangle$ is notoriously difficult, even in spherical symmetry 11]. This is even more true in our case, because the quantum state cannot be chosen among the standard ones, such as the Hartle-Hawking or Unruh states [12]. We shall therefore consider a simpler problem, that of quantizing fields in a two-dimensional version of the RNdeS spacetime. The metric is taken to be

$$
d s^{2}=-f d u d v
$$

where the null coordinate $u$ is defined by $d u=d v-$ $2 f^{-1} d r$. In two dimensions, the calculation of $\left\langle T_{a b}\right\rangle$ can be carried out explicitly [13]. For simplicity, we shall consider only the case of a conformally invariant scalar field. Below we will argue that the conclusions reached within the two-dimensional model should stay valid when applied to the four-dimensional spacetime.

We shall express $\left\langle T_{a b}\right\rangle$ in the coordinates $(u, v)$. However, we shall define the quantum state by expanding the scalar field into positive-frequency modes of the form $e^{-i \omega \bar{u}}, e^{-i \omega \bar{v}}$, where the transformations $\bar{u}(u)$ and $\bar{v}(v)$ will be specified shortly. In this state, and as given in Ref. [14], the renormalized expectation value of the stress-energy tensor is (we set $\hbar=1$ )

$$
\left\langle T_{a b}\right\rangle=\theta_{a b}+t_{a b}+(48 \pi)^{-1} R g_{a b} .
$$

Here, $R$ is the Ricci scalar associated with the twodimensional metric; $\theta_{a b}$ is a state-independent object whose non-vanishing components are

$$
\theta_{u u}=-(12 \pi)^{-1} f^{1 / 2} \partial_{u}^{2} f^{-1 / 2}
$$

and $\theta_{v v}$ (which is obtained by the replacement $u \rightarrow v$ ); $t_{a b}$ contains the information about the state and has nonvanishing components

$$
t_{u u}=(24 \pi)^{-1}\left[\frac{3}{2}\left(\bar{u}^{\prime \prime} / \bar{u}^{\prime}\right)^{2}-\bar{u}^{\prime \prime \prime} / \bar{u}^{\prime}\right]
$$

and $t_{v v}$ (obtained by the replacements $u \rightarrow v, \bar{u} \rightarrow \bar{v}$ ).

We need a quantum state that is regular at both $r=$ $r_{c}$ and $r=r_{e}$. Such a state has been constructed for the Schwarzschild-de Sitter spacetime by Marković and Unruh 15. It is trivial to generalize their construction to the RNdeS spacetime. The state is defined by choosing $\bar{u}$ to be an affine parameter along the null geodesic $v=$ $v_{0}=$ const., and $\bar{v}$ an affine parameter along the null geodesic $u=u_{0}$. The null geodesics intersect at a radius $r_{0}$ such that $r_{e}<r_{0}<r_{c}$. These choices imply

$$
\bar{u}^{\prime}=f\left[r\left(v_{0}-u\right)\right], \quad \bar{v}^{\prime}=f\left[r\left(v-u_{0}\right)\right],
$$


where we have indicated that $r$ is a function of $u$ and $v$, defined implicitly by $2 f^{-1} d r=d v-d u$.

A straightforward calculation, using the equations listed above, reveals that in the Marković-Unruh state, $\left\langle T_{a b}\right\rangle$ is given by

$$
\begin{aligned}
& \left\langle T_{u u}\right\rangle=-(48 \pi)^{-1}\left[F(u, v)-F\left(u, v_{0}\right)\right], \\
& \left\langle T_{u v}\right\rangle=(48 \pi)^{-1} f f^{\prime \prime} \\
& \left\langle T_{v v}\right\rangle=-(48 \pi)^{-1}\left[F(u, v)-F\left(u_{0}, v\right)\right],
\end{aligned}
$$

where

$$
F(u, v)=F[r(v-u)]=\frac{1}{4}\left(f^{\prime 2}-2 f f^{\prime \prime}\right) .
$$

For our purposes, the detailed functional form of $F(r)$ is not important. What is essential is that near a horizon $r=r_{j}, F(r)$ asymptotically behaves as

$$
F(r)=\kappa_{j}^{2}-\lambda_{j} f^{2}+O\left(f^{3}\right),
$$

where $\lambda_{j}=f^{\prime \prime \prime}\left(r_{j}\right) / 4 f^{\prime}\left(r_{j}\right)$ and $\kappa_{j}$ is the surface gravity. It can be checked that $\left\langle T_{a b}\right\rangle$ is regular at $r=r_{c}$ and $r=r_{e}$; this can be done by introducing well-behaved coordinates adapted to the horizon under consideration.

We now consider measurements made by observer $\mathcal{C}^{\prime}$. To remain static, this observer must be strongly accelerated. In the limit $f \rightarrow 0$, his scalar acceleration is given by $a \equiv\left(g_{a b} a^{a} a^{b}\right)^{1 / 2}=\kappa_{c} f^{-1 / 2}$, where $a^{a}=u_{; b}^{a} u^{b}$ is the acceleration vector. $\mathcal{C}^{\prime}$ is therefore immersed in a bath of thermal radiation (just as an accelerated observer in Minkowski spacetime would) with Unruh temperature $T_{c}=a / 2 \pi=\kappa_{c} f^{-1 / 2} / 2 \pi$ [16]. (Observer $\mathcal{C}$, who is freely falling, has no indication that this thermal bath exists.) The Unruh radiation is not all that the static observer sees, for (outgoing) quanta are also created in the vicinity of the black-hole past horizon. (These are also seen by $\mathcal{C}$.) These quanta are thermally distributed, and their locally-measured temperature $T$ varies according to Tolman's law, $T f^{1 / 2}=$ const. $=\kappa_{e} / 2 \pi 17$.

These conclusions are supported by the following result, obtained with the help of Eqs. (10) and (12). At late times $(u \rightarrow \infty)$, observer $\mathcal{C}^{\prime}$ measures an energy density given by (up to terms which are finite when $f \rightarrow 0$ )

$$
\left\langle\rho_{\mathcal{C}^{\prime}}\right\rangle=(48 \pi)^{-1} f^{-1}\left(\kappa_{e}{ }^{2}-\kappa_{c}{ }^{2}\right) .
$$

It is noteworthy that the Unruh radiation contributes negatively to $\rho_{\mathcal{C}^{\prime}}$. This can be understood as follows [18]. Consider an accelerated observer in Minkowski spacetime, performing measurements on a quantum field. We suppose that the field is in the true Minkowski vacuum state. The observer sees a thermal bath at temperature $a / 2 \pi$ [16], and associates to it an energy density $k(a / 2 \pi)^{n}$, where $k$ is a constant, $a$ the acceleration, and $n$ the dimensionality of spacetime. The observer also measures the vacuum polarization created by the quantum field. Because the renormalized energy density must be precisely zero if the field is in its true vacuum state, the vacuum polarization must contribute the negative amount $-k(a / 2 \pi)^{n}$ to the total result. This contribution does not depend on the quantum state, and is what appears as the second term to the right of Eq. (13).

The negative contribution associated with the vacuum polarization is present in every mode of the quantum field. Thus, in terms of measurements made by $\mathcal{C}^{\prime}$, the vacuum polarization cancels in the stress-energy tensor the contribution of the thermal flux emanating from the cosmological horizon. On the other hand, the thermal flux from the black-hole horizon is not cancelled (because of the difference in the horizon temperatures), and this results in the net (renormalized) flux (13), which is directed toward the future cosmological horizon. We note that $\mathcal{C}$ travels in the same direction as this flux and, due to redshift, measures a finite energy density. This accounts for the regularity of the stress-energy tensor at the cosmological horizon.

This partial cancellation by vacuum polarization explains how a steady value for $\left\langle\rho_{\mathcal{C}^{\prime}}\right\rangle$ can be compatible with a finite value of $\left\langle\rho_{\mathcal{C}}\right\rangle$ at the cosmological horizon. Contrary to the classical case, the redshift factor $e^{-2 \kappa_{c} v}$ needs not, and does not, appear in Eq. (13). [Recall the discussion following Eq. (3).] This is a key result, which has nothing to do with the fact that our expression for $\rho_{\mathcal{C}}$ was derived for a four-dimensional spacetime, while Eq. (13) holds in two dimensions. This result must be attributed to the different physics associated with the classical and quantum fields.

We are now ready to examine the behavior of the quantum stress-energy tensor in the vicinity of the Cauchy horizon. A slight difficulty is that the coordinates $(u, v)$ do not cover the portion of spacetime inside the black hole. To remedy this, we transform back to $v$ and $r$, using $2 f^{-1} d r=d v-d u$, which cover both the exterior and interior regions, up to $v=\infty$. For convenience, we then introduce the null coordinate $u^{\prime}$, which is defined only in the interior region, by the transformation $d u^{\prime}=2 f^{-1} d r-d v$. It is easy to see that under the combined transformation $(u, v) \rightarrow\left(u^{\prime}, v\right)$, Eqs. 110)-(12) keep the same form, except that $\left\langle T_{u^{\prime} v}\right\rangle$ comes with the opposite sign, and that $r$ is now a function of $u^{\prime}+v$.

We calculate $\left\langle\rho_{\mathcal{I}}\right\rangle$, the energy density as measured by observer $\mathcal{I}$. If $\kappa_{c} \neq \kappa_{i}$, we find

$$
\left\langle\rho_{\mathcal{I}}\right\rangle=\left(\tilde{E}^{2} / 48 \pi\right)\left(\kappa_{c}{ }^{2}-\kappa_{i}{ }^{2}\right) e^{2 \kappa_{i} v},
$$

which diverges in the limit $v \rightarrow \infty$. (The thermal flux, which emanates from the cosmological horizon and hits the observer head-on, is not cancelled by the local vacuum polarization.) If, on the other hand, $\kappa_{c}=\kappa_{i}$, then $\left\langle\rho_{\mathcal{I}}\right\rangle$ is regular at the Cauchy horizon. We conclude that the Cauchy horizon of a two-dimensional RNdeS black hole is quantum-mechanically unstable [19], except for the set of measure zero of spacetimes for which $\kappa_{i}=\kappa_{c}$.

We believe that this conclusion also applies to the fourdimensional spacetime. Indeed, it appears to us that the fundamental physics of the problem, which is revealed 
by the two-dimensional calculation, is robust and does not depend on the dimensionality of spacetime (or on the nature of the quantum field - its spin, conformality, etc.). Our results can all be intuitively explained in terms of fundamental processes such as the creation of thermal quanta near horizons, and the gravitational redshifts and blueshifts that these quanta undergo. (And once the contrast between $\rho_{\mathcal{C}^{\prime}}$ and $\left\langle\rho_{\mathcal{C}^{\prime}}\right\rangle$ is made, it follows easily from a blueshift argument that, generically, $\left\langle\rho_{\mathcal{I}}\right\rangle$ must diverge at the Cauchy horizon.) These processes take place equally well in four as in two dimensions 18. Physical intuition therefore suggests that our two-dimensional results should be qualitatively valid also in four dimensions.

We therefore conjecture that, excluding possibly a set of measure zero of such spacetimes, the Cauchy horizon of a four-dimensional RNdeS spacetime is always quantummechanically unstable. The mechanism for instability is the divergence of $\left\langle T_{\alpha \beta}\right\rangle$, the renormalized expectation value of the stress-energy tensor associated with quantized matter fields.

Conversations with R. Balbinot, K. Thorne, and W. Unruh were greatly appreciated. D.M. was supported by NSERC Grant 580441; E.P. was supported by NSF Grant AST 9114925.

[1] S.W. Hawking and G.F.R. Ellis, The large scale structure of space-time (Cambridge University Press, Cambridge, England, 1973).

[2] S. Chandrasekhar and J.B. Hartle, Proc. R. Soc. London A284, 301 (1982).

[3] W. Israel, Int. J. Mod. Phys. D 3, 71 (1994).

[4] R. Balbinot and E. Poisson, Phys. Rev. Lett. 70, 13 (1993).

[5] K.S. Thorne, in General Relativity and Gravitation 1992, Proceedings of the 13th International Conference on General Relativity and Gravitation, edited by R.J. Gleiser, C.N. Kozameh, and O.M. Moreschi (Institute of Physics Publishing, Bristol, England, 1993).

[6] B. Carter, in Black Holes, edited by C. DeWitt and B.S. DeWitt (Gordon and Breach, New York, 1973).

[7] F. Mellor and I. Moss, Class. Quantum Grav. 9, L43 (1992).

[8] P.R. Brady and E. Poisson, Class. Quantum Grav. 9, 121 (1992).

[9] C.M. Chambers and I. Moss, Class. Quantum Grav. 11, 1035 (1994).

[10] Here and below, the measured energy density is given by $\rho=T_{\alpha \beta} u^{\alpha} u^{\beta}$, where $u^{\alpha}$ is the observer's four-velocity. A similar statement holds for the quantum stress-energy tensor.

[11] See, e.g., P.R. Anderson, W.A. Hiscock, and D.A. Samuel, Phys. Rev. Lett. 70, 1739 (1993).

[12] W.A. Hiscock, Phys. Rev. D 39, 1067 (1989).

[13] P.C.W. Davies, S.A. Fulling, and W.G. Unruh, Phys. Rev.
D 13, 2720 (1976).

[14] R. Balbinot and R. Bergamini, Phys. Rev. D 40, 372 (1989).

[15] D. Marković and W.G. Unruh, Phys. Rev. D 43, 332 (1991).

[16] W.G. Unruh, Phys. Rev. D 14, 870 (1976).

[17] R.C. Tolman, Relativity, Thermodynamics, and Cosmology (Clarendon Press, Oxford, 1934).

[18] K.S. Thorne, R.H. Price, and D.A. Macdonald, Black Holes: The Membrane Paradigm (Yale University Press, New Haven, 1986) Chapter VIII.

[19] Here, we use the phrase "Cauchy horizon is unstable" as a synonym for "quantum stress-energy tensor diverges at the Cauchy horizon." Because no field equations have been supplied for the two-dimensional metric, the question of dynamical stability is not, strictly speaking, well posed. In four dimensions, however, the field equations are taken to be the semiclassical Einstein equations (in which $\left\langle T_{\alpha \beta}\right\rangle$ appears on the right-hand side), and the question of dynamical stability is well posed.

FIG. 1. Conformal diagram representing a portion of the RNdeS spacetime. Shown are the cosmological horizons at $r=r_{c}$ (future: $v=\infty$; past: $u=-\infty$ ), the black-hole outer horizons at $r=r_{e}$ (future: $u=\infty$; past $v=-\infty$ ), and the inner (Cauchy) horizon at $r=r_{i}$. Also shown are the asymptotic de Sitter region $r=\infty$, the timelike singularity $r=0$, and the world lines of our three observers. 
This figure "fig1-1.png" is available in "png" format from: http://arxiv.org/ps/gr-qc/9411002v1 